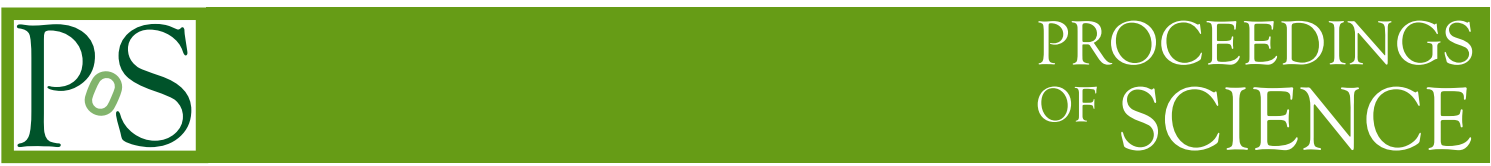

\title{
QCD physics measurements at the LHCb experiment
}

\section{Lorenzo Sestini on behalf of the LHCb collaboration ${ }^{a, *}$ \\ a INFN-Padova Division}

E-mail: lorenzo.sestini@cern.ch

The LHCb experiment offers a complementary phase space to ATLAS and CMS to study QCD processes, thanks to the forward acceptance and the large bandwidth of the trigger allowing low energy thresholds. For this reason $\mathrm{QCD}$ measurements at $\mathrm{LHCb}$ can provide unique constraints to the Parton Distribution Functions. Moreover these measurements can be used to probe perturbative QCD predictions, and to study hadronization processes. In these proceedings the latest QCD measurements that involve jets and performed during the Run II data taking are presented. In particular it is described how the measurement of the $Z+c$-jet production has been used to probe the instrinsic charm content of the proton.

*** The European Physical Society Conference on High Energy Physics (EPS-HEP2021), *** *** 26-30 July $2021 * * *$

*** Online conference, jointly organized by Universität Hamburg and the research center DESY ***

${ }^{*}$ Speaker 


\section{Introduction}

$\mathrm{LHCb}$ is a forward spectrometer, initially designed for $b$ and $c$ quarks physics [1]. Within the LHC experiments, $\mathrm{LHCb}$ alone provides precision coverage in the forward region of $p p$ collisions corresponding to the $2 \leq \eta \leq 5$ pseudo-rapidity range. In both LHC Run I and Run II LHCb demonstrated its capability in electroweak and QCD physics, qualifying it as a general purpose forward detector. Precision measurements of jets production and fragmentation are important tests of perturbative QCD and hadronization models. Moreover, the measurements of vector boson production in association with a jet can be used to probe Parton Distribution Functions (PDFs). At LHCb two different region are available in the $x-Q^{2}$ phase space, where $x$ is the momentum fraction of the parton and $Q^{2}$ is the transferred momentum: a region at low $x$, unexplored by other experiments, and a region at high $x$. The most recent QCD measurements performed by the LHCb experiment are presented in the next sections.

\section{Measurement of differential $b \bar{b}$ - and $c \bar{c}$-dijet cross-sections}

The production of $b$-jet and $c$-jet pairs in the forward region has been measured with $1.6 \mathrm{fb}^{-1}$ of integrated luminosity at $\sqrt{s}=13 \mathrm{TeV}$ [2]. In this analysis the differential $b \bar{b}$ - and $c \bar{c}$-dijet crosssections as a function of leading jet $p_{\mathrm{T}}$, leading jet $\eta$, rapidity difference and $m_{j j}$ are determined. The fiducial region of the measurement is defined by two jets with $p_{\mathrm{T}}>20 \mathrm{GeV} / c, 2.2<\eta<4.2$ and $\Delta \phi>1.5$. Reconstructed secondary vertices are required in both jets to select heavy-flavour. The sample selected in this way is formed by $b \bar{b}, c \bar{c}$ and background processes where one or two light jets are misidentified. The flavour composition is measured with a fit to the distribution of multivariate classifiers that have variables related to the secondary vertices as inputs. These observables are called $t_{0}$, for the light/heavy $(u d s g / b c)$ separation, and $t_{1}$, for the $b / c$ separation. The result of this fit is shown in figure 1. An unfolding technique is applied to correct for detector effects and measure the differential cross-sections, which are shown, as a function of $m_{j j}$, in figure 2 together with predictions. In the same figure the ratio of $c \bar{c}$ to $b \bar{b}$ cross-sections is also presented. The measurements are compatible with the predictions within the uncertainties, that are dominated by the systematic uncertainty on the heavy-flavour identification.

\section{Hadronization in Z-tagged jets}

The transverse momentum dependent jet substructure has been studied in $Z+$ jet events, where the $Z$ boson has been reconstructed in the dimuon final state. The Run I dataset at $\sqrt{s}=8 \mathrm{TeV}$, corresponding to $2 \mathrm{fb}^{-1}$ of integrated luminosity, has been used for this measurement [3]. Z+jet is the ideal system to study jet fragmentation since the amount of background is relatively low. Moreover this process is dominated by quark-initiated jets, and this is the first measurement of this kind in the forward region of $p p$ collisions and for jet $p_{\mathrm{T}}$ below $20 \mathrm{GeV}$. Jets are selected with $p_{\mathrm{T}}>10 \mathrm{GeV}$, and in the $2.5<\eta<4.0$ fiducial region. Three differential distributions for tracks inside the jets are measured:

- the longitudinal momentum fraction $z=\frac{\vec{p}_{\text {jet }} \cdot \vec{p}_{\text {hadron }}}{\left|\vec{p}_{\text {jet }}\right|^{2}}$, where $\vec{p}_{\text {jet }}$ and $\vec{p}_{\text {hadron }}$ are the jet and track momentum; 

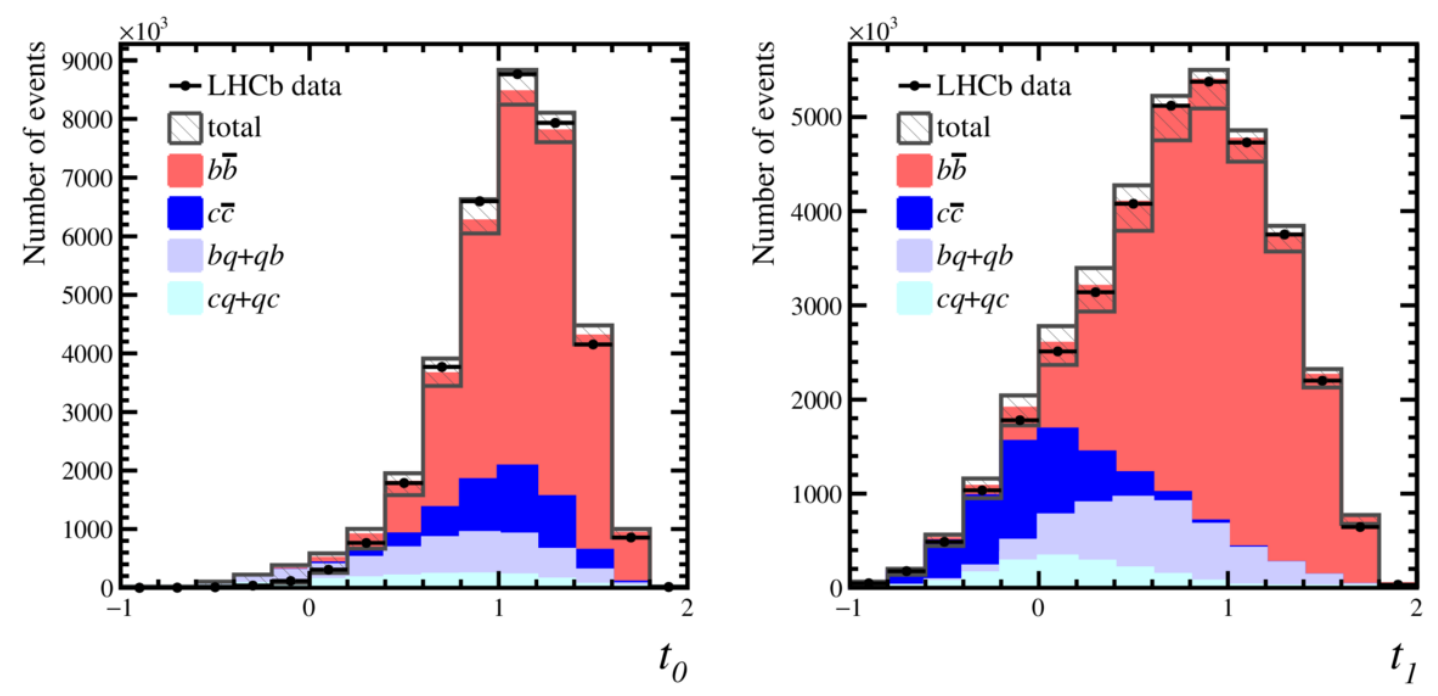

Figure 1: Result of the fit to the observables $\left(t_{0}, t_{1}\right)$, that are obtained with multivariate classifiers trained for the separation of $b, c$ and light jets.
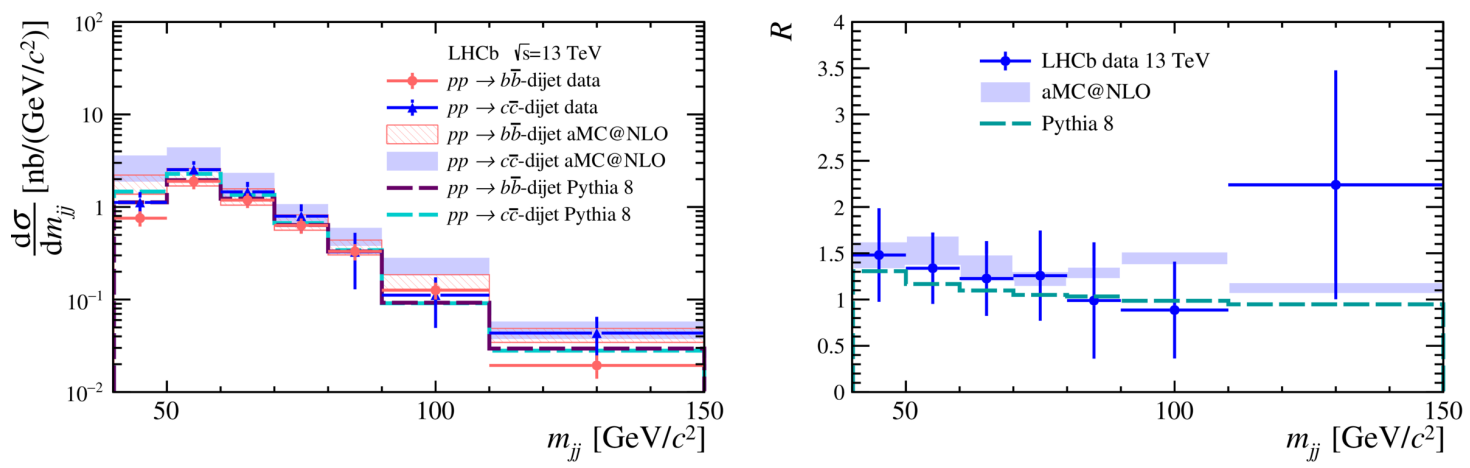

Figure 2: Left: differential $b \bar{b}$ - and $c \bar{c}$-dijet cross sections and $c \bar{c}$ to $b \bar{b}$ ratio as a function of $m_{j j}$. Right: ratio of $b \bar{b}$ to $c \bar{c}$ cross-sections as a function of $m_{j j}$.

- the transversal momentum fraction $j_{\mathrm{T}}=\frac{\vec{p}_{\mathrm{jet}} \times \vec{p}_{\text {hadron }}}{\left|\vec{p}_{\mathrm{et}}\right|^{2}}$

- the radial distance $r=\sqrt{\left(\phi_{\text {jet }}-\phi_{\text {hadron }}\right)^{2}+\left(y_{\text {jet }}-y_{\text {hadron }}\right)^{2}}$, where $\phi_{\text {jet }}\left(y_{\text {jet }}\right)$ and $\phi_{\text {hadron }}\left(y_{\text {hadron }}\right)$ are the azimuthal angle(rapidity) of jet and track respectively.

The distributions are corrected for jet and track efficiencies, and a 2-dimensional unfolding technique is applied on $\left[z, p_{\mathrm{T}}^{\mathrm{jet}}\right],\left[j_{\mathrm{T}}, p_{\mathrm{T}}^{\mathrm{jet}}\right]$ and $\left[r, p_{\mathrm{T}}^{\mathrm{jet}}\right]$ distributions to correct for detector resolution effects. The distributions are also normalized to the number of selected $Z+$ jet events. The results are shown in figure 3 for three different $p_{\mathrm{T}}^{\text {jet }}$ ranges. These results can be compared with the outcome of jet fragmentation generators, and eventually they can be used to tune them. 

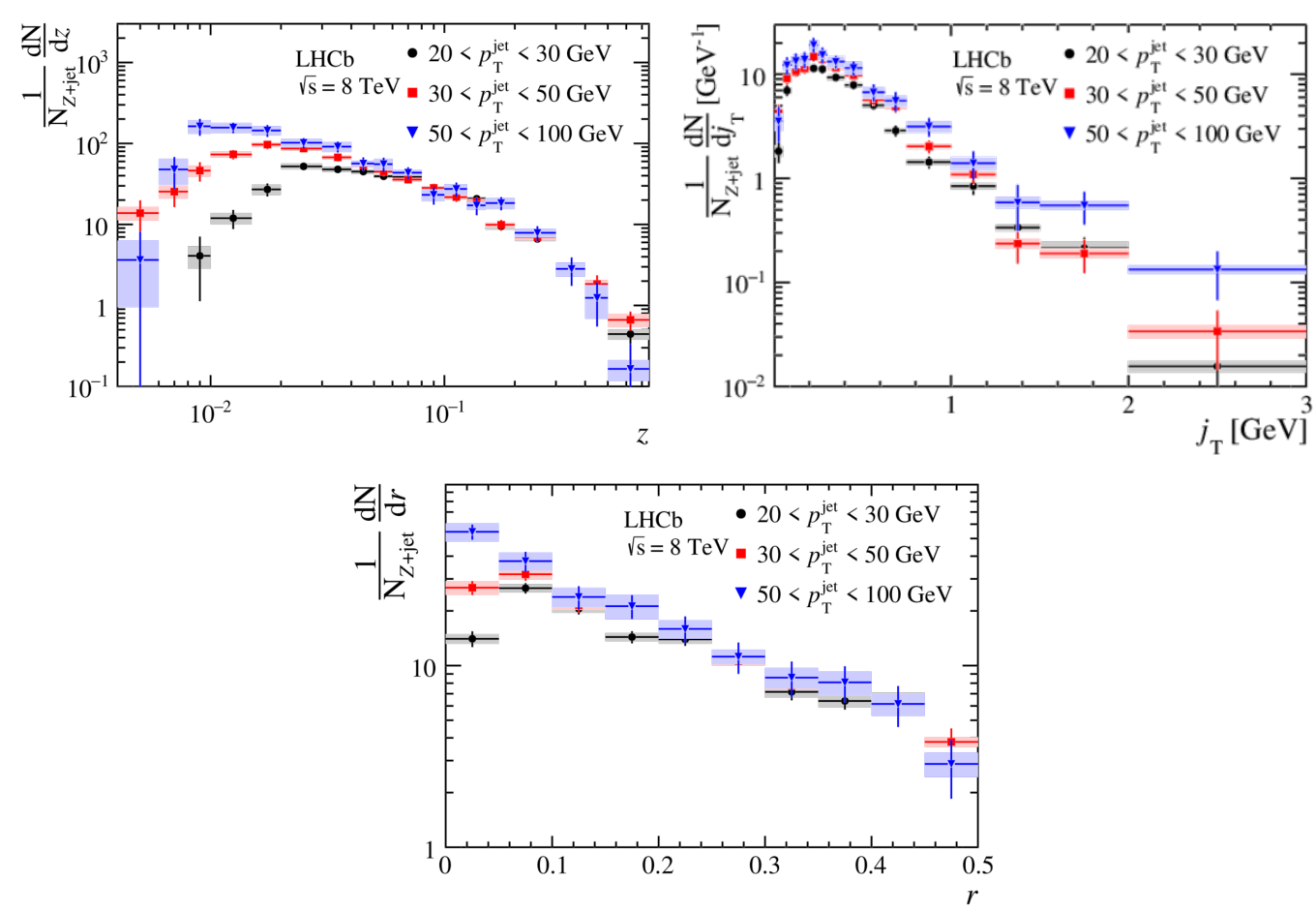

Figure 3: Measured charged hadron distributions in Z-tagged jets, for $z, j_{\mathrm{T}}$ and $r$.

\section{Intrinsic charm in $Z+$ jet events}

In proton content charm can be extrinsic, if it is produced by the gluon splitting $g \rightarrow c \bar{c}$, or intrinsic, if it is present in a bound state with the valence quark, like $\mid u u d c \bar{c}>$. The PDF of the instrinsic charm can be sea-quark like or valence-quark like, and the latter is predicted by the Light Front QCD theory (LFQCD). In particular, the high- $x$ region of the charm PDF is sensitive to the intrinsic charm component. The intrinsic charm is ruled out at $1 \%$ by previous measurements, but lower contributions are still possible. The $Z+c$-jet system is particularly interesting as probe of the instrinsic charm. As shown in figure 4 , the $Z+c$-jet to the $Z+$ jet cross section ratio, $\frac{\sigma(Z c)}{\sigma Z j}$, as a function of the jet rapidity is sensitive to the intrinsic charm component, especially at high rapidities. For this reason the $\mathrm{LHCb}$ experiment is the ideal place to study it.

The full $\sqrt{s}=13 \mathrm{TeV}$ dataset has been used for this measurement, for a total of $6 \mathrm{fb}^{-1}$ of integrated luminosity [4]. The $Z$ boson is reconstructed in the dimuon final state and displaced vertex (DV) is required inside the jet cone. A fit to the DV-mass and the number of tracks in the DV is performed to extract the flavour component of the data sample, its result is shown in figure 5. The templates used in this fit are obtained from heavy-flavour jets enriched data samples. The fitted yields are corrected for selection efficiencies and unfolded for detector resolution effects. The dominant uncertainty is related to the $c$-tagging efficiency, that is obtained from calibration data samples. The measurement of $\frac{\sigma(Z c)}{\sigma Z j}$ as a function of $\mathrm{Z}$ boson rapidity is shown in figure 6 . In the interval of rapidity between 3.5 and 4.5 , the intrinsic charm hypothesis is compatible with the measurement, while the no intrinsic charm hypothesis is $3 \sigma$ away. However the measurement 


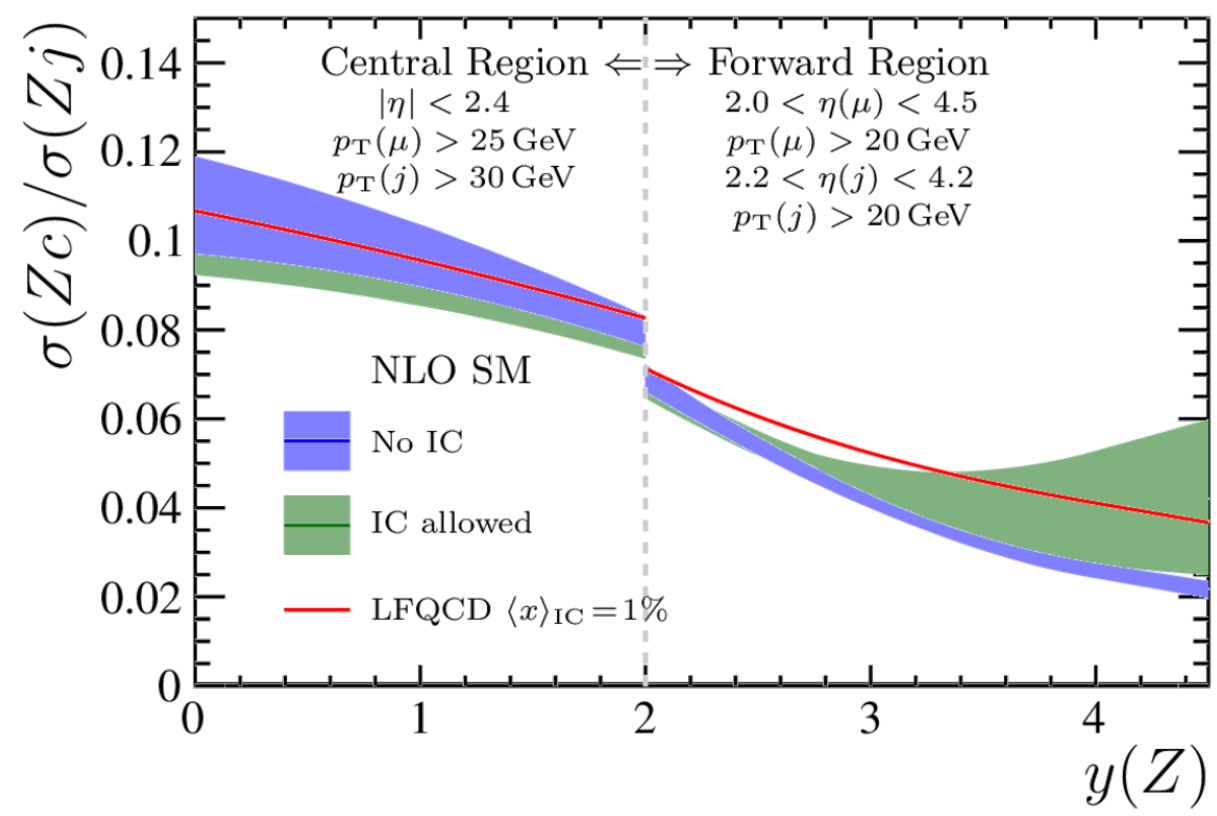

Figure 4: $\frac{\sigma(Z c)}{\sigma Z j}$ ratio as a function of the $Z$ boson rapidity, obtained with different predictions that may include or not the intrinsic charm component of the proton [4].

is dominated by the statistical uncertainty, therefore more data are needed to reach a conclusive statement. This will be possible by studying the events that will be collected in the Run III data taking.
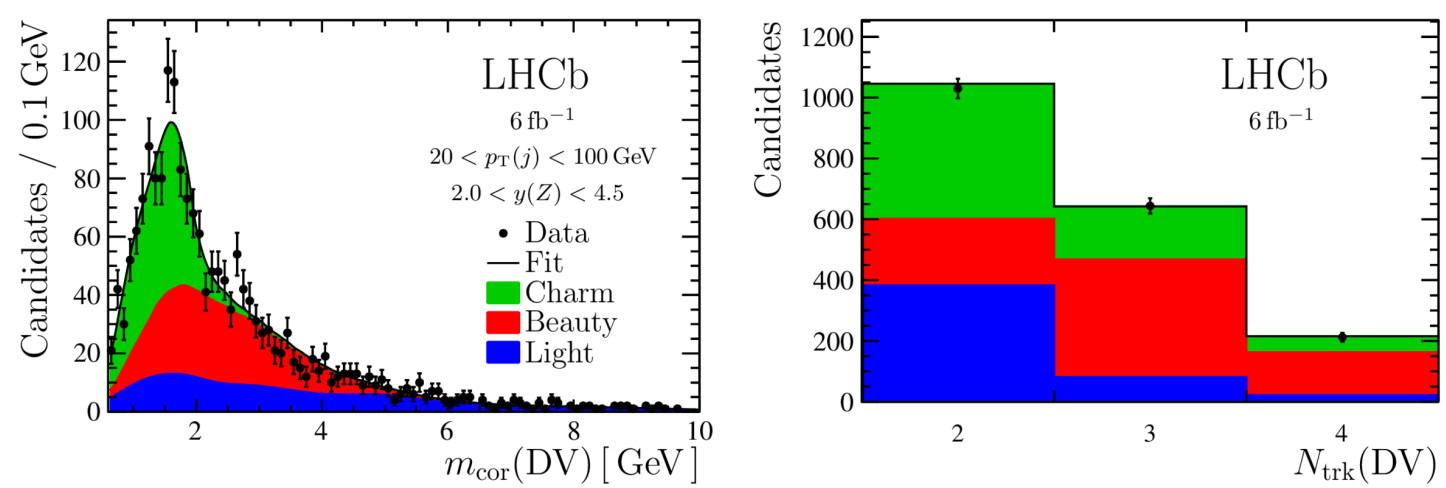

Figure 5: Result of the fit to the displaced-vertex mass (left) and number of tracks (right) distributions of $Z+c$-jets candidates.

\section{Conclusions}

In this document the most recent QCD measurements performed by studying $p p$ collisions at $\mathrm{LHCb}$ have been presented. The measurement of the $b \bar{b}$ - and $c \bar{c}$-dijet cross sections provide 


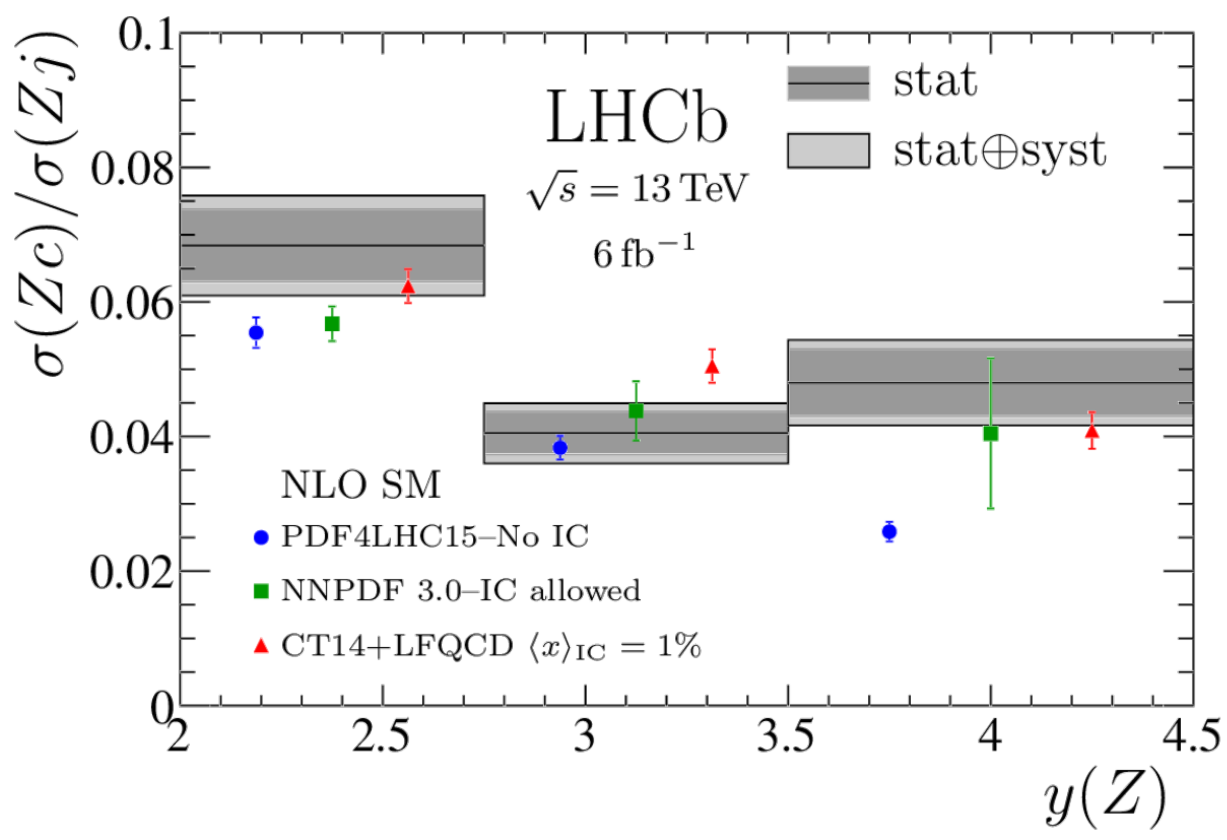

Figure 6: Measurement of $\frac{\sigma(Z c)}{\sigma Z j}$ as a function of $Z$ boson rapidity, compared with predictions obtained from different models, that may include or not the intrinsic charm component.

a valuable test of perturbative QCD in the forward region, while the measured charged hadrons distributions in $Z$-tagged jets can be used to study fragmentation and tune Monte Carlo generators. The measurement of the $Z+c$-jet system has given a hint of intrinsic charm component in the proton, that should be confirmed by collecting more data.

\section{References}

[1] A. A. Alves Jr. et al., The LHCb detector at LHC, JINST 3 (2008) S08005.

[2] LHCb Collaboration, Measurement of differential $b \bar{b}$ - and $c \bar{c}$-dijet cross-sections in the forward region of pp collisions at $\sqrt{s}=13 \mathrm{TeV}$, JHEP 02 (2021) 023

[3] LHCb Collaboration, Measurement of charged hadron production in Z-tagged jets in protonproton collisions at $\sqrt{s}=8 \mathrm{TeV}$, Phys. Rev. Lett. 123, 232001 (2019)

[4] LHCb Collaboration, Study of Z bosons produced in association with charm in the forward region, arXiv:2109.08084 